\title{
Photon Beamlines and Diagnostics at LCLS
}

S. Moeller ${ }^{1 \star}$, J. Arthur ${ }^{1}$, A. Brachmann ${ }^{1}$, R. Coffee ${ }^{1}$, F.-J. Decker ${ }^{1}$, Y. Ding ${ }^{1}$, D. Dowell ${ }^{1}$, S. Edstrom $^{1}$, P. Emma ${ }^{1}$, Y. Feng ${ }^{1}$, A. Fisher ${ }^{1}$, J. Frisch ${ }^{1}$, J. Galayda ${ }^{1}$, S. Gilevich ${ }^{1}$, J. Hastings ${ }^{1}$, G. Hays $^{1}$, P. Hering ${ }^{1}$, Z. Huang ${ }^{1}$, R. Iverson ${ }^{1}$, J. Krzywinski ${ }^{1}$, S. Lewis ${ }^{1}$, H. Loos ${ }^{1}$, M.

Messerschmidt ${ }^{1}$, A. Miahnahri ${ }^{1}$, H.-D. Nuhn ${ }^{1}$, D. Ratner ${ }^{1}$, J. Rzepiela ${ }^{1}$, D. Schultz ${ }^{1}$, T. Smith ${ }^{1}$, P.

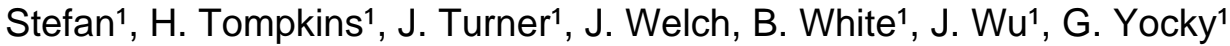

R. Bionta2, E. Ables², B. Abraham², C. Gardener², K. Fong², S. Friedrich², S. Hau-Riege'2, K. Kishiyama², T. McCarville², D. McMahon², M. McKernan², L. Ott², M. Pivovaroff'2, J. Robinson², D. Ryutov², S. Shen², R. Soufli2

G. Pile 3

1SLAC National Accelerator Laboratory, Stanford, CA 94309, USA

2Lawrence Livermore National Laboratory, Livermore, CA 94550, USA

${ }^{3}$ Argonne National Laboratory, Argonne, IL 60439, USA

*Corresponding author: smoeller@slac.stanford.edu

Abstract: The LCLS hard x-ray Free Electron Laser at SLAC reported first lasing in April of 2009. Since then two successful user runs have been completed at the two soft $\mathrm{X}$-ray stations. The first hard x-ray station has started commissioning in July of 2010. Beam diagnostics play an essential role for tuning the machine and delivering the requested beam properties to the users. An overview of the LCLS photon diagnostics will be presented including some selected commissioning results. Plans for future improvements and upgrades will be briefly discussed.

Keywords: $x$-ray free electron laser; $x$-ray photon diagnostics; solid attenuator; gas attenuator; $\mathrm{x}$-ray imager; pulse-energy detector; $\mathrm{x}$-ray mirrors

\section{Introduction}

The Linac Coherent Light Source (LCLS) is the first hard-x-ray free electron laser in operation [1]. The turn-on of LCLS was rapid and operation has been reliable. Performance has exceeded the design parameters in several areas. The photon energy output covers a range from $480 \mathrm{eV}$ to over $9 \mathrm{keV}$; the pulse energy is typically $2-3 \mathrm{~mJ}$, with a maximum of $4 \mathrm{~mJ}$ at $2 \mathrm{keV}$. Electron pulse lengths can be varied from 500 fs to shorter than $10 \mathrm{fs}$. A low-charge option at $20 \mathrm{pC}$ is being explored, which delivers pulses shorter than 10 fs with a reduced pulse energy, typically around $0.2 \mathrm{~mJ}$. On-demand, single-shot and multi-shot modes up to $60 \mathrm{~Hz}$ (planned is $120 \mathrm{~Hz}$ ) can be made available.

The photon diagnostics built for LCLS have been commissioned and provide measurements of various properties of the FEL beam, such as pulse energy, beam size and position, wavelength, and allows for intensity attenuation over the entire wavelength range. 
The two soft X-ray instruments, the Atomic Molecular and Optics (AMO) and Soft X-ray Material Science (SXR) stations, are fully operational and completed their second user run in mid September 2010. The third user run is scheduled from October to December 2010, and will include the first hard x-ray instrument X-ray Pump-and-Probe (XPP). Three additional hard xray stations will follow: CXI (Coherent X-ray Imaging) is planned to start commissioning in December 2010, the XCS (X-ray correlation spectroscopy) instrument will start in June 2011, and the station for Matter in Extreme Conditions (MEC) in 2012. A list of past and future milestones for LCLS commission and operations is shown in table 1.

\section{LCLS Overview}

The LCLS Free-Electron-Laser uses the last $1 \mathrm{~km}$ of the $3 \mathrm{~km}$ existing LINAC at SLAC, in addition to about $800 \mathrm{~m}$ of new construction, which houses electron beam transport, the undulator, the diagnostic front end and two experimental halls connected by a $200 \mathrm{~m}$ long photon transport tunnel. For the accelerator layout and first lasing results see reference [1].

The off-axis injector with its photocathode RF gun and amplified Ti:Sapphire laser creates an electron bunch of typically $0.25 \mathrm{nC}$ with a 35 A peak current and an transverse emittance of less than $1 \mu \mathrm{m}$ (RMS). After injection of the electron bunch into the straight path of the LINAC, three accelerator structures produce electron kinetic energies of up to $13.6 \mathrm{GeV}$. Two bunch compressors shorten the electron bunch to create a 100 fold increase in peak current to $3.5 \mathrm{kA}$. A transverse-deflection cavity, located after the second compressor, allows electron pulse length measurements as short as $10 \mathrm{fs}$. Several feedback loops are implemented to control and stabilize the beam. For example, by controlling the peak current, it is possible to directly control the electron pulse length and therefore the x-ray pulse length [2].

The undulator is $132 \mathrm{~m}$ long and consists of 33 segments. Each segment consists of a $3.4 \mathrm{~m}$ long planar permanent magnet which has a period of $3 \mathrm{~cm}$ and a fixed gap of $6.8 \mathrm{~mm}$ with a magnetic field of 1.25 Tesla, resulting in a k-value of 3.5. The undulators and focusing magnets sit on a girder system that allow for vertical and horizontal control of five degrees of freedom to submicron level. Each of the 33 segments is fully retractable on its girder system. Alignment of the undulator in respect to the electron beam is achieved through a beam based alignment method that measures the electron trajectory at various energies. The girder positions are continuously monitored to an accuracy of $<1 \mu \mathrm{m}$ with a stretched wire position monitoring and a hydrostatic leveling system. Commissioning showed that saturation at $8 \mathrm{keV}$ could be achieved with only 23 undulator segments inserted which is now the typical configuration during operation.

After the electron beam dump the FEL beam enters the separate Front End Enclosure (FEE) which houses a suite of components to prepare and characterize the beam before it enters the experimental stations in the Experimental Halls. The lay-out of the photon beam lines is shown in figure 1. 
A system of grazing-incidence x-ray mirrors, located at the downstream end of the FEE, splits the beam into soft and hard x-ray branches and distributes the beam into a total of six experimental hutches; 3 in each of the experimental halls. The soft x-ray branch splits again into the AMO beam line in hutch 1 and the SXR beamline ending in hutch 2 . The SXR beamline also contains a transmission chamber in hutch 1 . The hard X-ray line directs the beam to the XPP station in hutch 3 of the Near Hall and to the 3 Far Hall experiments: the X-ray Correlation Spectroscopy (XCS) instrument in hutch 4, the Coherent X-ray Imaging (CXI) instrument in hutch 5, and the station for Matter in Extreme Conditions (MEC) in hutch 6. A laser room, equipped with a Ti:Sapphire system is located in the Near Hall, with a laser transport pipe leading to each of the 3 hutches for conducting pump-probe experiments.

\section{LCLS Photon Diagnostics}

The main photon diagnostics for LCLS is located in the FEE, a 30m long, separate tunnel located downstream of the electron dump area. The photon diagnostics were designed and fabricated by Lawrence Livermore National Laboratory (LLNL).

The primary function of the photon diagnostics is to measure, control and transport the beam for use in the experimental areas. The diagnostics in the FEE were specified for identifying and tuning the FEL during commissioning, as well as to characterize and optimize beam properties during operations. It consists of absorbers, imagers and intensity monitors. A downstream view of the FEE is shown in figure 2. In addition, there is an instrument-specific diagnostic suite located upstream of each of the hard x-ray instruments, which permits collimation, attenuation, pulse selection and focusing of the beam as well as measurement of beam profile, intensity and position. The commissioning of the instrument-specific hard $\mathrm{x}$-ray diagnostic package is underway and will be described elsewhere.

Figure 3 shows a schematic layout of the diagnostic and optical components in the FEE. The FEL beam and the spontaneous radiation enter unobstructed through the $25 \times 45 \mathrm{~mm}$ wide opening of the fixed mask into the FEE. Due to the high peak power of the FEL beam, most materials would suffer damage due to melting. Extensive damage studies [3] have shown that boron carbide $\left(\mathrm{B}_{4} \mathrm{C}\right)$ is a suitable candidate to withstand power densities at this location and therefore a layer of $\mathrm{B}_{4} \mathrm{C}$ is used to protect all collimators, slits, stoppers and soft $\mathrm{x}$-ray mirror surfaces. Pop-in cameras are located throughout the beam transport to monitor beam positions and spot size.

\subsection{X-ray Slit System}

The first upstream component in the FEE is the x-ray slit, which consists of a four-jaw slit system distributed along the beam direction. It is fully adjustable, from an aperture of $25 \mathrm{~mm} \mathrm{x}$ $45 \mathrm{~mm}$ to completely closed. Each slit features a $10 \mathrm{~mm}$ thick $\mathrm{B}_{4} \mathrm{C}$ plate bonded onto a $50 \mathrm{~mm}$ tungsten alloy block. Figure 4 shows the beam at various apertures. 


\subsection{Attenuator System}

The attenuator system is a combination of a pressurized gas pipe and solid beryllium disks of various thicknesses, which together provide attenuation of $10^{4}$ over the energy range from 800 to $8000 \mathrm{eV}$ (see figure 5). The gas attenuator is a $4.5 \mathrm{~m}$-long nitrogen-gas-filled pipe with controllable gas pressure of up to 12 Torr for use in the low energy range (up to $2000 \mathrm{eV}$ )[4]. It is flanked upstream and downstream by transition sections with $4 \mathrm{~mm}$-diameter differentiallypumped $\mathrm{B}_{4} \mathrm{C}$ apertures mounted into gate valves, which allow the $800 \mathrm{eV}$ FEL with a beam size of about $1 \mathrm{~mm}$ (FWHM) to pass unobstructed. The aperture system masks allow for collinear alignment with the beam axis. The two differential pumping sections are independent mechanical systems, which are isolated by bellows and are equipped with four-degree-of-freedom, motorized alignment movers.

The solid attenuator provides attenuation at higher energies above $2 \mathrm{keV}$, by inserting up to $7 \mathrm{Be}$ windows of various thickness $(0.5$ (2), 1, 2, 4, 8, and $16 \mathrm{~mm})$. This configuration supports thicknesses from 0 to $32 \mathrm{~mm}$ in $0.5 \mathrm{~mm}$ steps and provides at least 3 steps for every decade of attenuation. The $\mathrm{B}_{4} \mathrm{C}$ apertures and $\mathrm{Be}$ attenuators are fully retractable, so that the clear aperture is defined by the larger upstream fixed mask. Two thin Be filters (IF1 Be foil) 10 and $100 \mu \mathrm{m}$ thick, are added to block visible light especially at lower photon energy settings.

The attenuation was measured by comparison between the upstream and downstream gas detectors, illustrated in Figure 6, as a function of the beryllium thickness, at a photon energy of $8.3 \mathrm{keV}$. The measured attenuation length of $3.73 \mathrm{~mm}$ is close to the calculated design value of $4.2 \mathrm{~mm}$. Some degradation of FEL beam images was observed when using the Be attenuators, which was attributed to the quality of the Be. An upgrade is planned for the near future to correct this. The gas attenuator performs reliably in the soft $\mathrm{x}$-range and still achieves an attenuation of $10^{3}$ at photon energies as high as $1500 \mathrm{eV}$.

\subsection{Gas Energy Detector}

Two gas detectors located in the differential pumping sections of the gas attenuator, provide nonintrusive FEL energy measurement on a pulse-by-pulse basis [5]. The gas detector was not designed to provide an absolute intensity measurement, but was intended to be calibrated with an absolute device such as the total energy monitor (see 3.4). The gas detectors measure the $\mathrm{N}_{2}$ fluorescence produced by the FEL beam entering a $30 \mathrm{~cm}$ long and $8 \mathrm{~cm}$ diameter gas chamber which is pressurized between 0.02-1.2 Torr. The aluminum chamber walls are grooved to suppress any stray light entering the two perpendicularly-installed photomultiplier tubes. Magnetic coils around the chamber confine the photoelectrons and maximize the fluorescence signal. The nitrogen ionization leads to the emission of photo - and Auger electrons, which deposit their energy in the nitrogen gas until they thermalize or collide with the chamber walls. The exited $\mathrm{N}_{2}$ molecules relax by fluorescence in the near UV, between 300-400 $\mathrm{nm}$. The fluorescence yield in air and nitrogen has been studied in detail for the detection of highly- 
energetic cosmic rays and it was found that the fluorescence yield per deposited energy is only weakly dependent on the energy of the exciting electron [6]. Processes such as interaction with the walls, quenching at higher pressures and space charge effects at different FEL energies and operating pressures may affect the performance and are being studied [7].

In conjunction with the photon-based diagnostics another energy measurement of the FEL beam has been developed. It determines the energy loss that electrons suffer in the undulator as they are kicked on the various orbits leading to varying FEL power. This method has been automated and optimized and gives reliable values for the average pulse energy in a matter of only a few minutes. An example of such an energy loss scan is shown in figure 7 (left) and a correlation plot with the gas detector signal in figure 7 (right), which has a relative error typically between 1 to $5 \%$ (RMS).

The pulse-by-pulse energy at $8.3 \mathrm{keV}$, measured with the gas detector and accumulated over 1000 pulses, is shown in Figure 8. The pulse-energy jitter is about 8\% (RMS).

\section{4. $K$-monochromator}

The so called " $K$-monochromator", located downstream of the attenuator system, consists of two remotely-insertable, channel-cut silicon crystals and transmits only a single photon energy of 8.2 $\mathrm{keV} \mathrm{[8].} \mathrm{With} \mathrm{the} \mathrm{ability} \mathrm{to} \mathrm{remotely} \mathrm{insert} \mathrm{and} \mathrm{retract} \mathrm{single} \mathrm{undulator} \mathrm{segments,} \mathrm{the} K$ monochromator has been successfully used to characterize the $K$-value of undulator segments relative to each other or in comparison to a reference segment. Measurements determining the content of higher harmonics in the spectrum have also been a valuable diagnostic tool.

\subsection{Total Energy Monitor}

The Total Energy Monitor (TEM) measures the total energy of each pulse based on the temperature rise in a $0.5 \mathrm{~mm} \mathrm{Si} \mathrm{substrate.} \mathrm{It} \mathrm{is} \mathrm{a} \mathrm{destructive} \mathrm{measurement} \mathrm{(beam} \mathrm{intercepting)}$ and measures the pulse energy in real time on a pulse-by-pulse basis. The sensor material, mounted on the back side, is made from rare-earth-manganites, also known as colossal magnetoresistive (CMR) materials $[9,10]$. The device is designed such that the temperature rise and response of the material allows for single-pulse measurements over a dynamic range of $2 \mathrm{~mJ}$. The sensor is cooled with a pulse tube refrigerator (Vericold Technologies) and internally calibrated by a pulsed optical laser $(532 \mathrm{~nm})$ and built-in attenuator. The internal laser pulse meters are absolutely calibrated to $\pm 5 \%$ and determine the expected absolute error of the TEM. Five different sensor masks (TEM \#1-5) are used to minimize variations due to beam jitter and varying beam sizes.

The internal laser calibration demonstrates the very linear response of the bolometer. Figure 9 shows an example of a correlation between the output signal of the TEMs with the FEL and the gas detector, calibrated via electron energy loss, over the range of the solid attenuator. In this case, both measurements agree within an error of about $6 \%$. However, reproducibility of the 
internal calibration and signal stability over longer time scales has not been adequate to obtain a stable absolute calibration. Further studies are planned for the future.

\subsection{Direct Imager}

The last device in the FEE before the offset mirror system is the Direct Imager. It consists of two electronic imaging systems that view a set of scintillators mounted on a remotely-moveable shaft. Two thicknesses of the YAG:Ce scintillators are provided: a $5 \mu \mathrm{m}$ crystal for the soft x-ray range below $2 \mathrm{keV}$ and a $50 \mu \mathrm{m}$ crystal for the hard x-ray range. In addition, a $1 \mathrm{~mm}$ thick crystal can be used for imaging very low intensities, e.g. the spontaneous radiation of single undulator segments in combination with the k-monochromator. The optics of the wide-field-of-view (WFOV) imager provides $117 \mu \mathrm{m}$ resolution, and is optimized for the larger beam sizes in the soft x-ray range while the narrow-field-of-view (NFOV) has $20 \mu \mathrm{m}$ resolution and is better suited for the smaller spot sizes in the hard x-ray. Both imaging systems are equipped with highspeed CCD cameras (Cascade 512B) with 20 fps and neutral-density filter wheels to avoid saturation, mounted outside the vacuum. Camera focus and the filter wheels are remotely controlled.

The Direct Imager is one of the main diagnostic tools at LCLS. An example of an FEL beam image with fitted line shapes for the intensity distribution, is shown in figure 10. The beam size was consistently larger than predicted and believed to be due to the smaller electron beam emittance and thus larger divergence of the photon beam. The integrated intensity can be related to the beam energy and was measured as a function of the solid attenuator beryllium thickness (see figure 11). Saturation is noticeable above $170 \mu \mathrm{J}$, whereas other high-order effects and noise contribute to the signal below $2.5 \mu \mathrm{J}$. The pulse energy was $1.4 \mathrm{~mJ}$ for figure 10 .

\subsection{Offset Mirror Systems}

In the downstream half of the FEE tunnel, a set of grazing-incidence mirrors (see figure 3 for the location of the Offset Mirrors) deflects the FEL beam to the various experimental stations and also removes higher-energy spontaneous undulator radiation and bremsstrahlung background from the spectrum. An arrangement of collimators shields the scattered high-energy radiation from passing downstream. A total of four soft x-ray mirrors (SOMS), $250 \mathrm{~mm}$ long, direct the beam horizontally into the soft x-ray branch and distribute the beam to either the AMO or SXR instrument. When the first SOMS mirror is not inserted, the photon beam enters the hard X-ray beamline and is offset horizontally by a pair of $450 \mathrm{~mm}$ long hard x-ray offset mirrors (HOMS).

These are silicon-carbide-coated and have a cut-off at $25 \mathrm{keV}$, to permit reflection of the FEL $3^{\text {rd }}$ harmonic for an $8.3 \mathrm{keV}$ fundamental. The soft x-ray offset mirrors (SOMS) are boron-carbidecoated and have a cut-off at $2 \mathrm{keV}$. Preserving the transverse coherence of the beam puts high demands on the surface figure and finish requirements. Substrate fabrication, thin film deposition, precision metrology and mounting resulted in figure errors on the order of $2 \mathrm{~nm}$ (RMS) $[11,12]$. The effect of the SOMS and HOMS mirrors on the wavefront has been 
calculated using surface metrology data [13]. Some of the offset and experimental station x-ray mirrors started to show signs of varying degrees of discoloration after several weeks of operation. Surface degradation is not due to mechanical damage but, as chemical analysis showed, due to carbon deposition presumably accumulating over time from imperfect vacuum conditions combined with the extremely high brightness of the LCLS beam. Possible solutions to address this issue are currently being discussed.

\section{Summary and Future Plans}

The x-ray diagnostics instrumentation of the LCLS provides various ways of measuring and controlling the FEL beam to support machine tuning and user operations. Beam commissioning results demonstrated overall good performance. Areas for improvement have also become apparent and are already being addressed. As the user demand for delivering beams with specific properties increase, machine capabilities and diagnostic tools need to be developed to match this demand. One of the goals will ultimately include the pulse-by-pulse analysis of as many beam properties as possible, particularly the beam size, pulse length, absolute pulse energy and also measurement and control of the FEL spectrum, coherence and polarization.

With the rapidly-rising number of proposals for LCLS science and the completion of all six instruments expected by 2012, increasing available user access time is becoming very important. A short-term plan is to implement beam-sharing methods to allow parallel x-ray beam use at several instruments at the same time. A long-term plan is being developed to build a second injector that could serve soft and hard x-ray undulators independently.

\section{Acknowledgements}

We are grateful for the support of the US Department of Energy, Office of Science, under contract no. DE-AC02-76SF005. This work was performed under the auspices of the U.S. Department of Energy by Lawrence Livermore National Laboratory under Contract DE-AC5207NA27344.

\section{References:}

[1] P. Emma et al., Nature Photonics 4, 641-647, (2010)

[2] H. Loos, PAC09, Vancouver, British Columbia, Canada, TU3GRI01, (2009)

[3] S. Hau-Riege et al. accepted for publication in Optics Express.

[4] D. Ryutov et al., LCLS Technical Note, LCLS-TN-09-5, (2008)

[5] S. Hau-Riege et al., J. Appl. Phys. 103, 053306, (2008)

[6] M. Nagano et al., Astropart. Phys. 22, 235, (2004) 
[7] S. Hau-Riege et al. PRL 105, 043003, (2010)

[8] J. Welch et al., FEL2009, Liverpool, UK, THOA05, (2009)

[9] G.J Yong et al., Sensor Letters vol. 6, 741-745, (2008)

[10] O.B. Drury et al., IEEE Trans. Act. On Nuclear Science, Vol.56, No. 3, (2009)

[11] R. Soufli et al., Proc. SPIE 7077, 707716-1, (2008)

[12] R. Soufli et. al. Proc. SPIE 7361, 73610U (2009)

[13] A. Barty et al., Optics Express, Vol. 17, No. 18, 15508, (2009) 


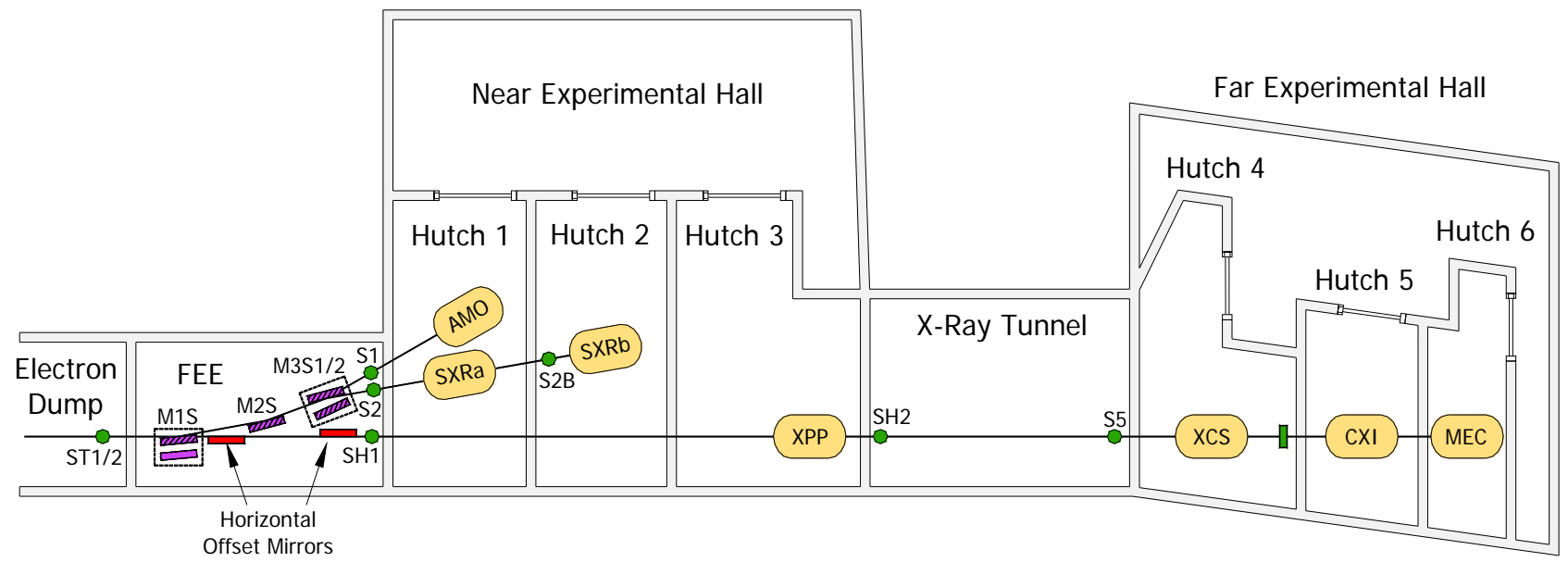

Fig. 1: LCLS Photon beam line layout with instrument stations.

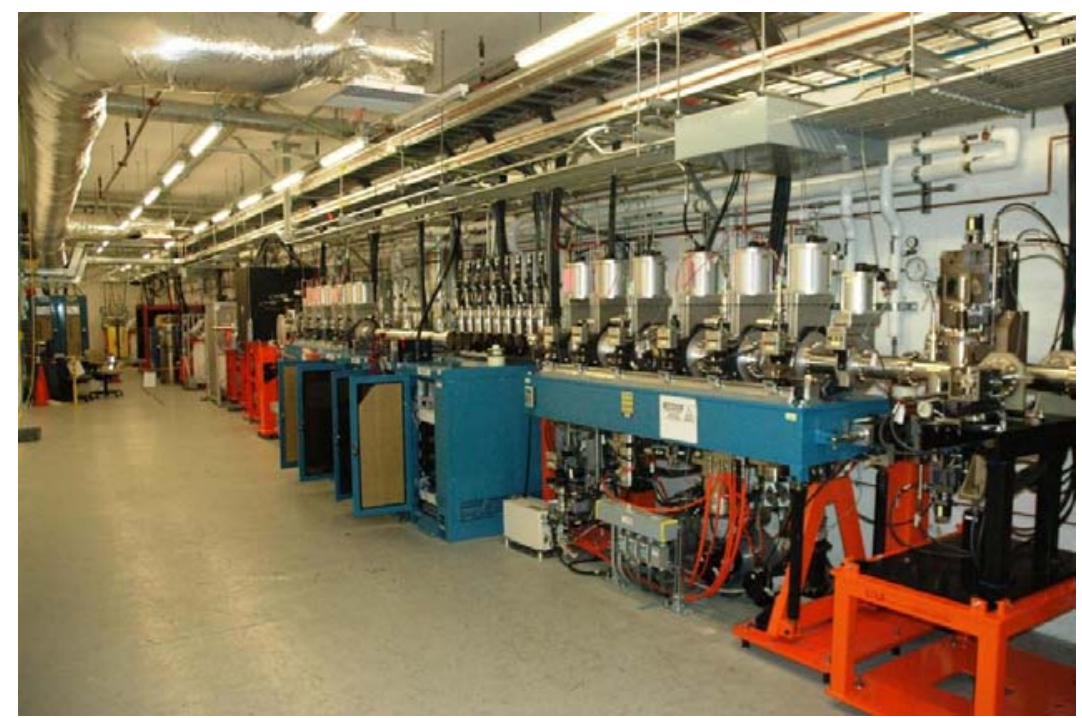

Fig. 2: Downstream view of the FEE. From the right: Slit system, attenuator system, total energy monitor (black cube) and the mirror system in the background. 


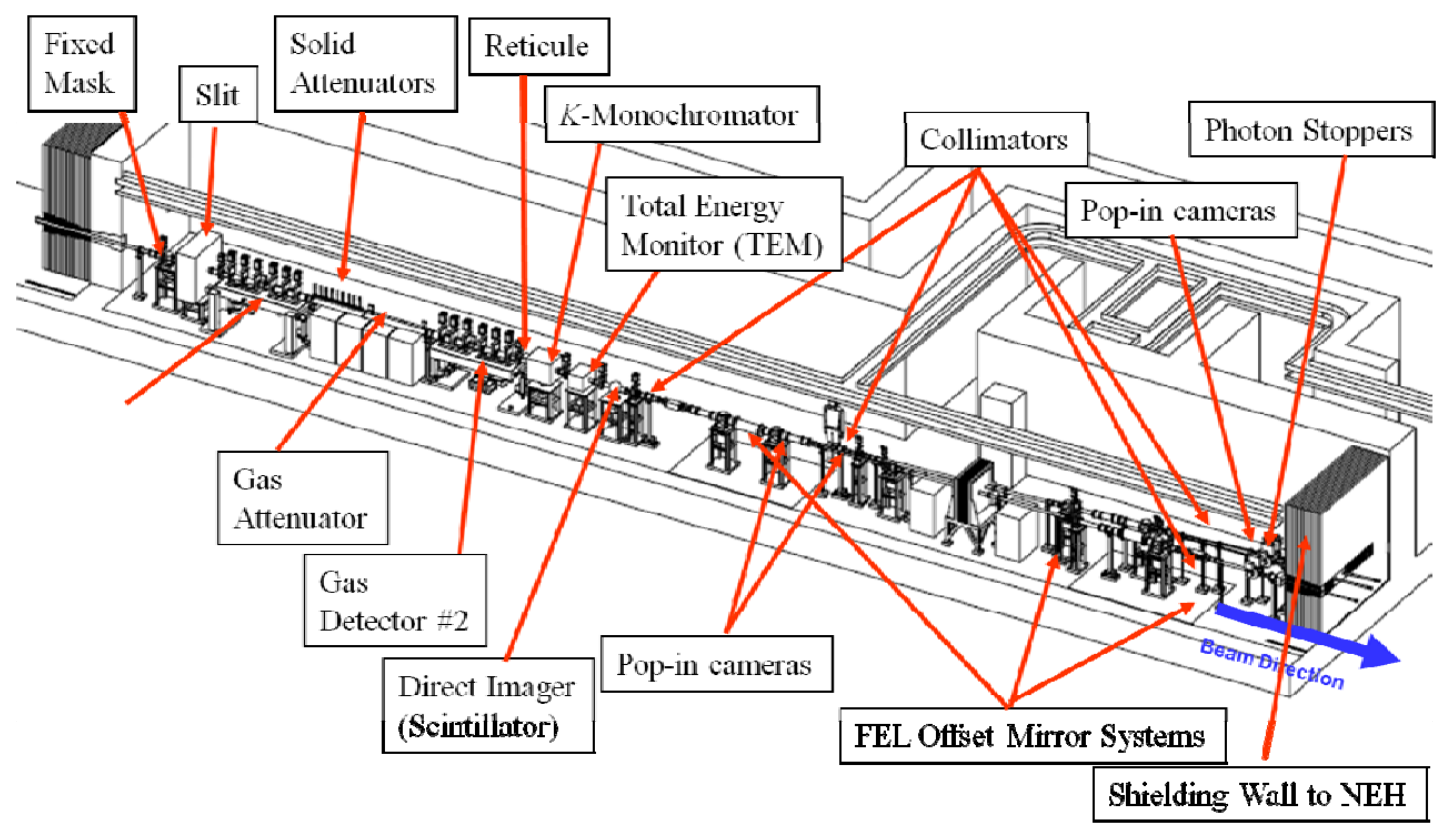

Fig .3: Layout of the FEE with diagnostic and optical components.
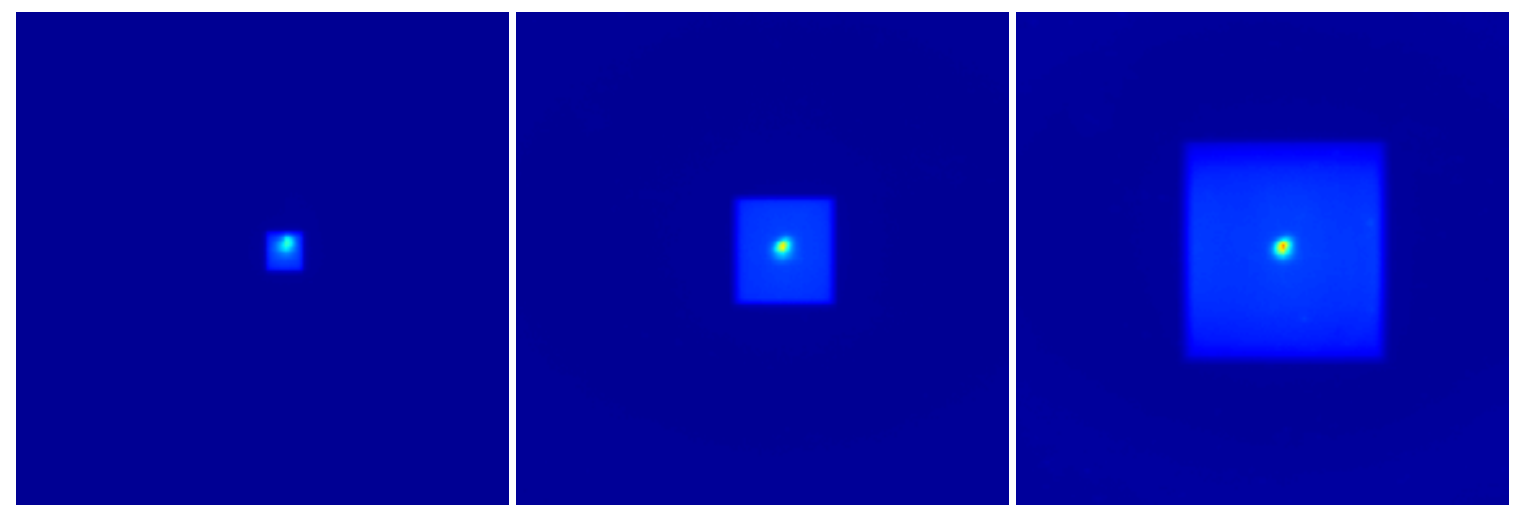

Fig. 4: Images (taken using the Direct Imager) of the $8.3 \mathrm{keV}$ beam at slit positions of 2, 5, and $10 \mathrm{~mm}$ (image size is $20 \times 20 \mathrm{~mm}$ ). 


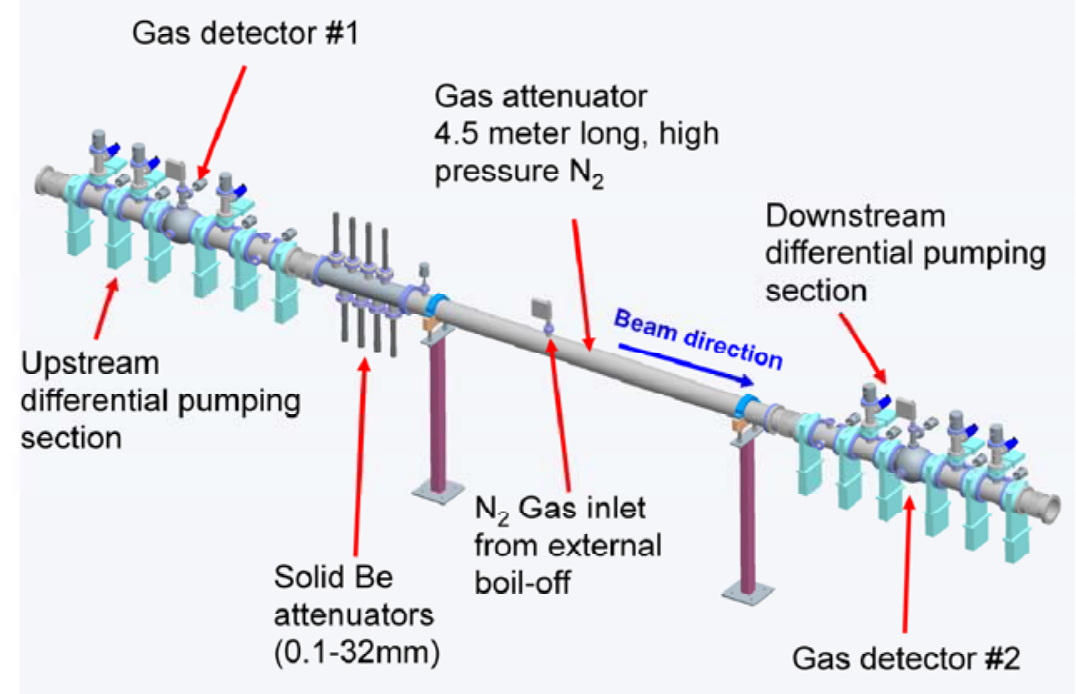

Fig. 5: LCLS attenuator system: Gas and solid attenuators with differential pumping sections and integrated gas detector monitors.

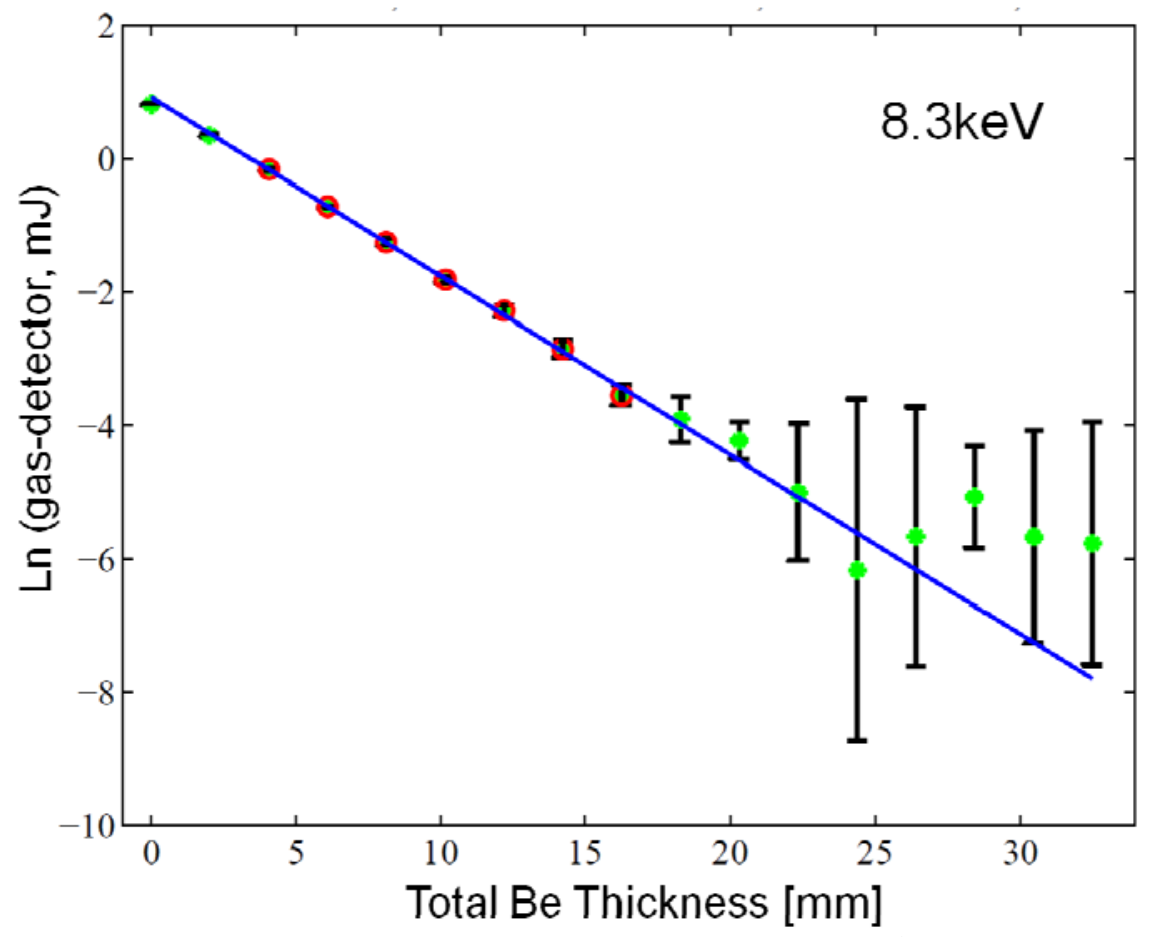

Fig. 6: Solid attenuator performance measured with the Gas Detectors as a function of beryllium attenuator thickness at $8.3 \mathrm{keV}$. 

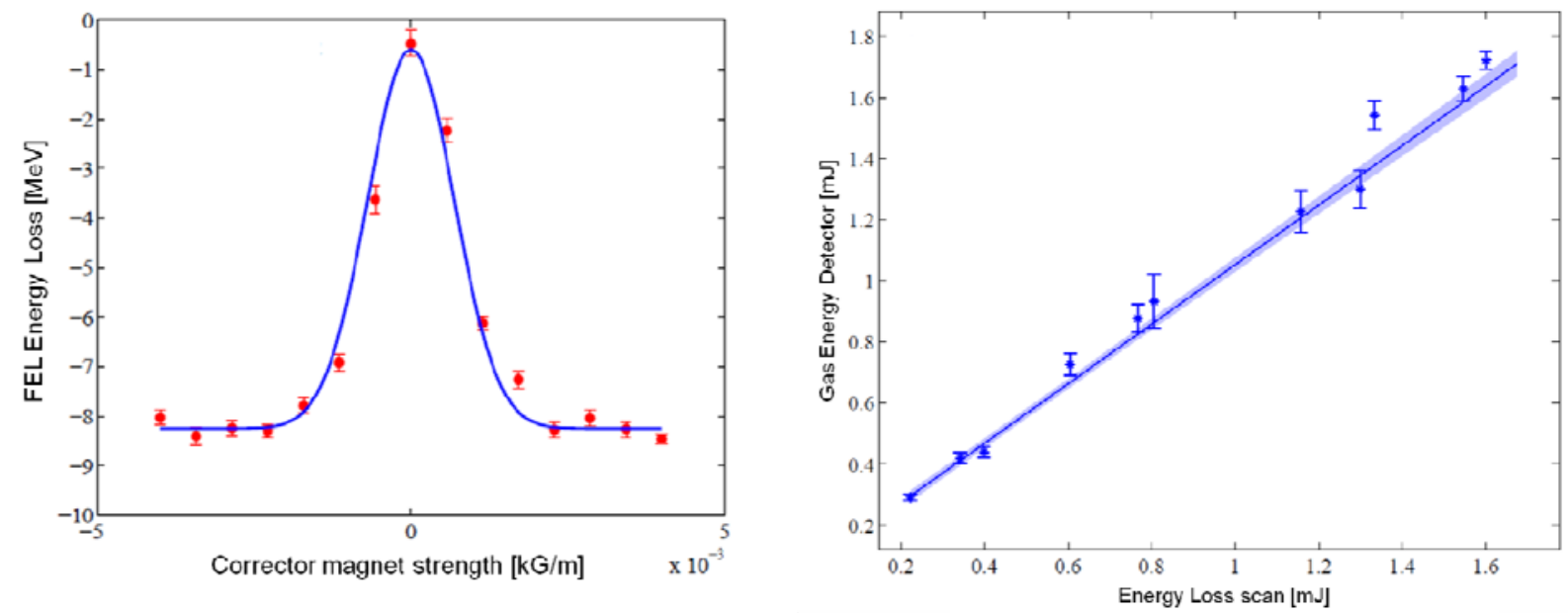

Fig. 7: Electron energy loss scan (left) and correlation with the gas detector signal (right) at $8.3 \mathrm{keV}$.

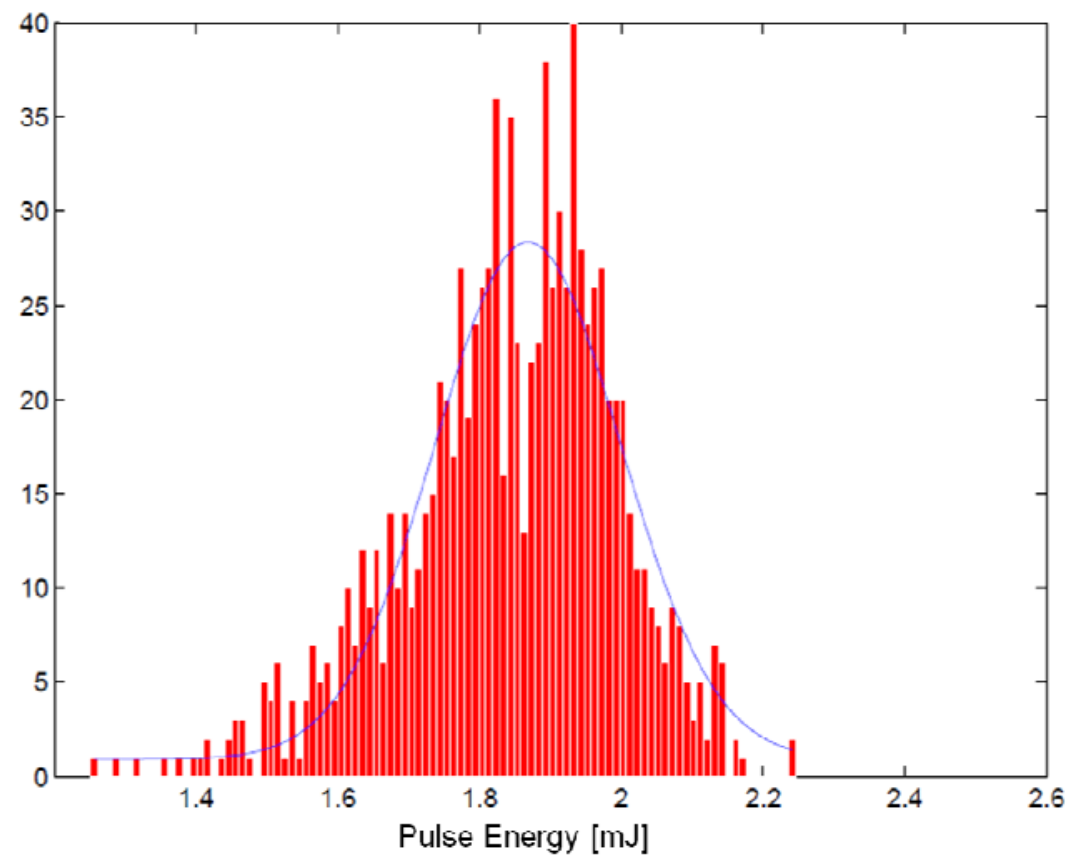

Fig. 8: Pulse energy histogram measured with the gas detector at $8.3 \mathrm{keV}$. 


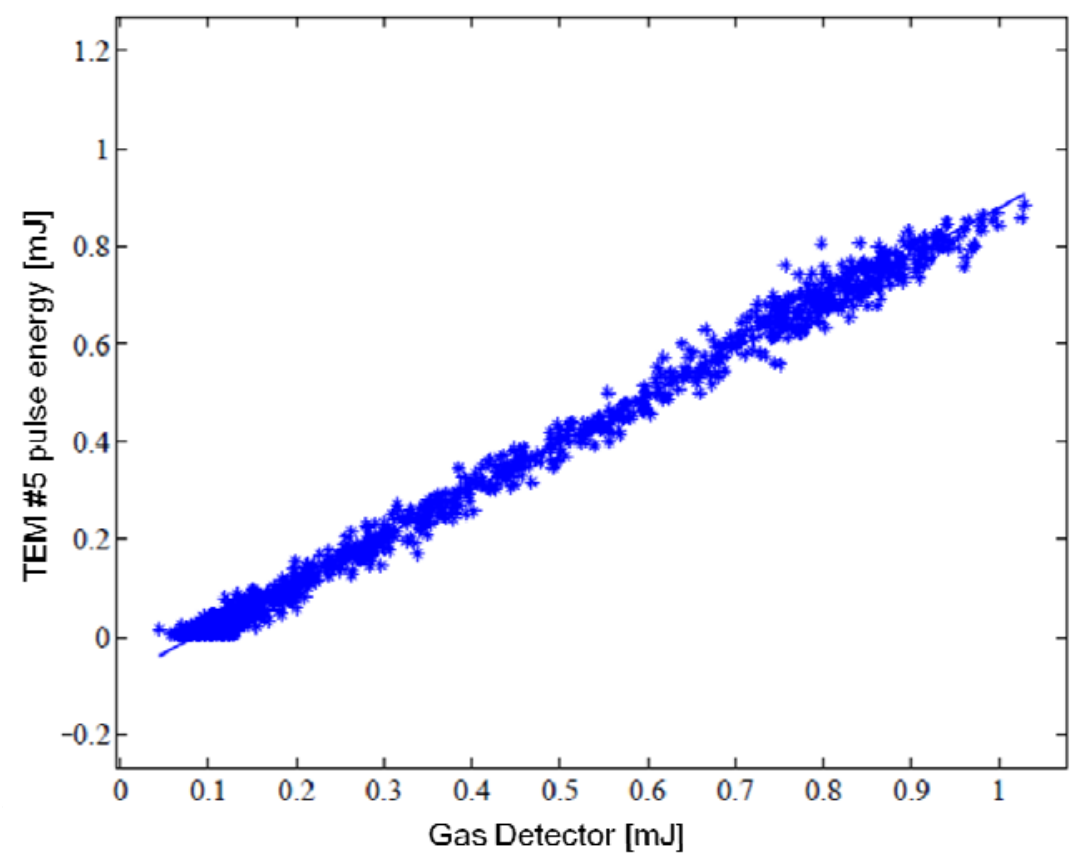

Fig. 9: Correlation plot of the Total Energy Monitor signal versus gas detector signal as a function of beryllium attenuator thickness.

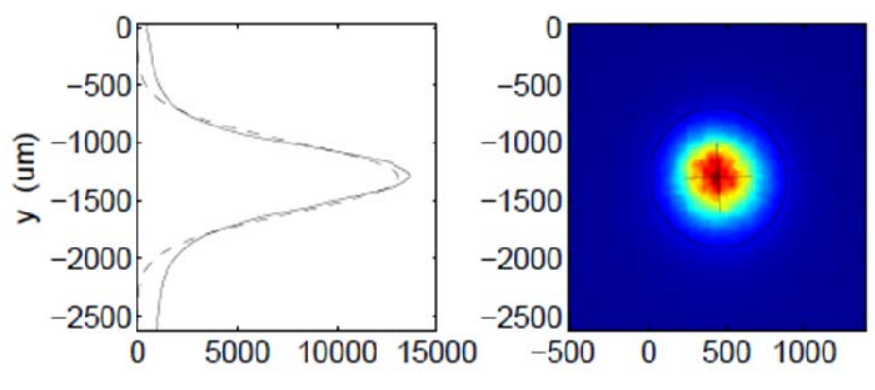

$$
\begin{aligned}
& \text { xmean }=445.40 \text { um } \\
& \text { ymean }=-1298.76 \text { um } \\
& \text { xrms }=218.00 \text { um } \\
& \text { yms }=301.56 \text { um } \\
& \text { corr }=-0.03 \\
& \text { sum }=9.92 \text { Mcts }
\end{aligned}
$$

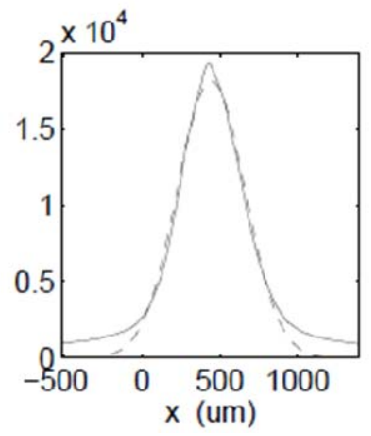

Fig. 10: LCSL beam image recorded by the Direct Imager System, with fits of the intensity line shapes. 


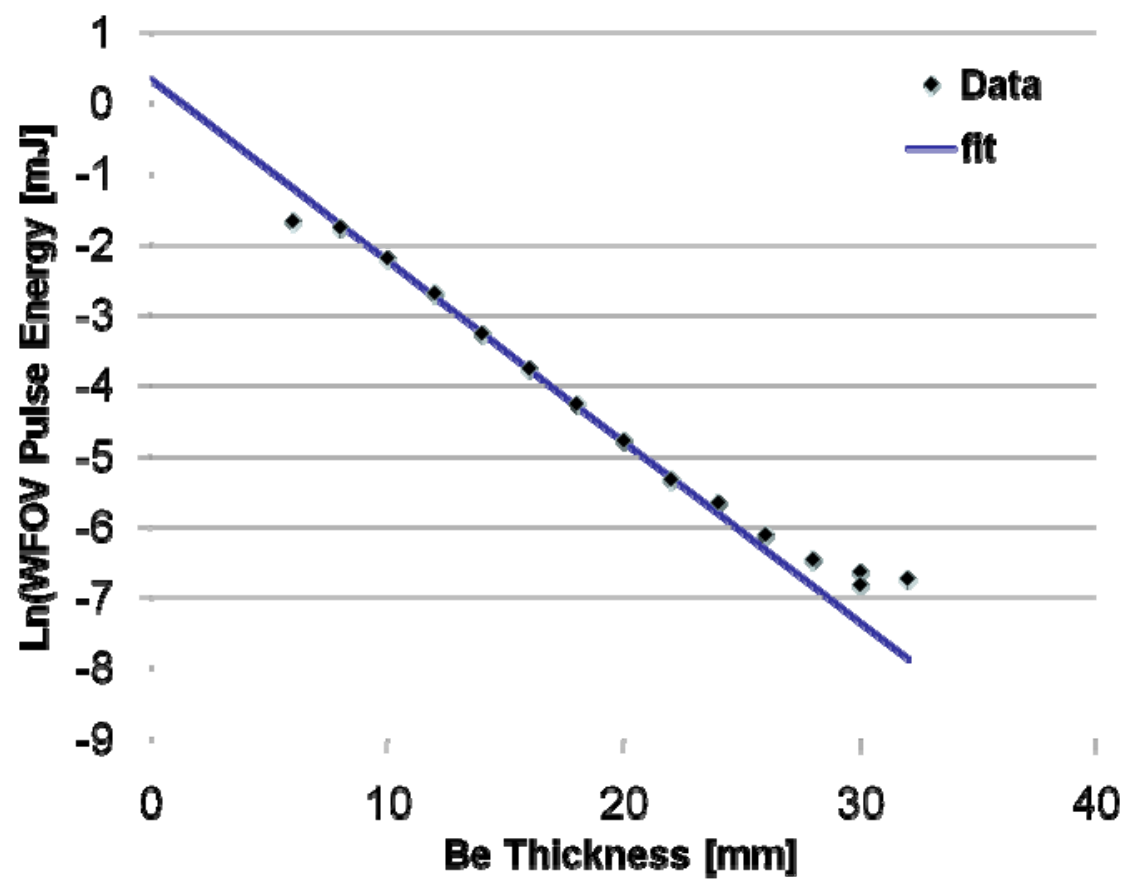

Fig.11: Direct Imager (WFOV) measured pulse energy as a function of beryllium attenuator thickness. The measured Be attenuation length differs by about $9 \%$ from the theoreticallyexpected value.

\begin{tabular}{|l|l|}
\hline Laser gun commissioned & 2007 \\
\hline Linac and bunch compressors commissioned & 2008 \\
\hline First electron beam through undulator & $12 / 13 / 2008$ \\
\hline 21 undulator magnets installed and ready & $4 / 7 / 2009$ \\
\hline First lasing at 1.5A & $4 / 14 / 2009$ \\
\hline X-ray diagnostics commissioned & July-Sept. 2009 \\
\hline Soft x-ray in NEH AMO instrument hutch 1 & $8 / 18 / 2009$ \\
\hline Start of user operations & $11 / 1 / 2009$ \\
\hline SXR commissioning/ $2^{\text {nd }}$ user run & May 2010 \\
\hline XPP commissioning start & June 2010 \\
\hline $3^{\text {rd }}$ user run AMO/SXR/XPP & Sept. - Dec. 2010 \\
\hline CXI commissioning start & Dec. 2010 \\
\hline XCS commissioning start & June 2011 \\
\hline MEC commissioning start & 2012 \\
\hline
\end{tabular}

Table 1: LCLS Milestones for commissioning and operation. 